\title{
Accountabilty y Mejoramiento Educativo: análisis de experiencias internacionales
}

\author{
Luis Felipe de la Vega Rodríguez' \\ 'Pontificia Universidad Católica de Chile, Santiago - Chile
}

RESUMEN - Accountabilty y Mejoramiento Educativo: análisis de experiencias internacionales. Este artículo analiza diferentes formas en que el mecanismo de accountability ha sido incorporado en las políticas educacionales de un grupo de países, como estrategia para el mejoramiento educativo. Para cada caso de estudio, se realizó un análisis del diseño, nivel de presencia de este mecanismo y su uso para promover mejoramiento. El análisis comparado mostró que solo algunos casos cuentan con tipologías puras de accountability. El resto, ha incorporado otras estrategias y principios educativos en sus diseños de políticas, o retrocedió en la centralidad que había entregado accountability como apuesta para favorecer el mejoramiento educativo.

Palabras clave: Accountabilty Educacional. Mejoramiento Educativo. Educación Comparada. Política de la Educación.

ABSTRACT - Accountability and Educational Improvement: analysis of international experiences. This article discusses various ways in which accountability mechanism has been incorporated into the educational policies of a group of countries as a strategy for educational improvement. A study on the design and the level of presence of this mechanism as a strategy that seeks to promote educational improvement was made. The comparative analysis of the cases showed that only some of them have pure types of educational accountability. The remaining cases have incorporated other strategies and educational principles into their policies or reversed on the centrality that was given to accountability, as strategy to promote educational improvement.

Keywords: Educational Accountability. Educational Improvement. Comparative Education. Educational Policy.

Educação \& Realidade, Porto Alegre, v. 40, n. 1, p. 275-298, jan./mar. 2015.

275

Disponível em: <http://www.ufrgs.br/edu_realidade> 


\section{El Mejoramiento Educativo: desafío y objetivo de los sistemas educacionales}

El concepto de mejoramiento educativo se vincula con un cuerpo de investigación con más de treinta años de historia, dirigidos al análisis y evaluación de políticas e innovaciones educativas que buscan alcanzar mejoras en los procesos y/o resultados educativos. Su enfoque ha profundizado en la idea de mejora como un proceso que involucra a las personas e instituciones que participan de su implementación, preguntándose sobre qué tiene que ocurrir para que se introduzca efectivamente en un escenario educativo, considerando variables externas e internas a la escuela, materiales y simbólicas (Fullan, 1982; 2002). De esta forma, se ha buscado establecer conexiones entre la investigación y las políticas educativas, para generar conocimiento que favorezca su diseño e implementación (Murillo, 2003; Fullan, 2002; Hopkins, 2008).

Existe consenso respecto a que los procesos de mejoramiento debieran tener como foco central la relación entre enseñanza y aprendizaje (Fullan, 2011; Hopkins, 2008; Levin, 2009). Elmore (2010) denomina a esta relación como núcleo pedagógico, compuesto por la interacción dentro del aula entre el docente, el estudiante y los contenidos. Las dinámicas y acciones que resulten de esa interacción se relacionarán directamente con el nivel de aprendizaje y la calidad educativa.

Otro elemento relevante en el proceso de mejoramiento es que debiera contar además con la condición de sustentabilidad, lo que implica una lógica progresiva y gradual de instalación (Hargreaves; Fink, 2006; Hopkins, 2008; Barber, 2010). En esa línea, Murillo y Krichesky (2012) sostienen que la literatura sobre mejoramiento escolar ha ido consolidando la idea de que este proceso ocurre a través de fases, las que no son lineales, puesto que las decisiones presentes van afectando las futuras y en algunos casos ayudan a avanzar, mientras en otras provocan retrocesos.

El estudio del mejoramiento educativo ha priorizado en la última década orientaciones hacia las que debieran dirigirse las políticas educativas, indicando focos de interés para la investigación. Hargreaves (2010) indica que las reformas debieran orientarse hacia la flexibilidad, el desarrollo de la colaboración y confianza dentro de la escuela, el respeto por las diferencias y la profundidad, por sobre la estandarización (Hargreaves; Shirley, 2012).

Respecto del proceso de enseñanza- aprendizaje, el rol de los docentes y de los estudiantes, Darling-Hammond (2004; 2006; 2010) señala que la necesidad de trabajar con niños de diferentes características, diversos aprendizajes complejos y aplicados, presiona a la pedagogía y a las políticas a apoyar la enseñanza de gran calidad y apuntar al aprendizaje de alto nivel. En la misma línea, Hargreaves (2003; 2010) estima que los procesos de mejoramiento debiesen orientarse hacia que los 
estudiantes desarrollen habilidades que le permitan responder a los requerimientos de lo que el autor denomina como sociedad del conocimiento, donde son relevantes los atributos de flexibilidad y creatividad.

\section{El Accountability, como Estrategia de Mejoramiento}

Dentro de las estrategias de política educativa que se utilizan para promover el mejoramiento educativo, el accountability en sistemas educativos es un mecanismo que ha sido implementado en variados sistemas educativos y que además ha estado bajo un intenso proceso de análisis y debate, sobre sus principios, orientaciones y resultados (Hopkins, 2008; McBeath, 2011).

Desde un punto de vista general, Möller (2009) y CLAD (2000) señalan que el accountability como mecanismo incluye dos componentes: responder o responsabilizarse por las propias acciones y además rendir cuentas por lo que ocurrió con el área de propia actuación. En el campo educacional, el accountability cuenta con estructuras y sentidos que pueden organizarse y operar desde tipologías disímiles. Respecto de éstas, Darling-Hammond y Ascher (1991) señalan que algunas de ellas comparten funciones más amplias, vinculadas a todo ámbito de gestión pública. Tal es el caso del accountability político, asociado a la labor de los gobernantes y representantes en el proceso eleccionario, y el legal, en que los ciudadanos pueden recurrir a la justicia, si se transgrede alguna normativa sobre el funcionamiento educativo.

Por su parte, se identifican tres grandes tipos de accountability específicamente educativos (Darling-Hammond, 2004). El primero es el accountability burocrático, orientado por el desafío de la equidad, a través del cual los actores educativos deberán asegurar el cumplimiento de las normas, programas educativos y otras prácticas hacia todos los estudiantes.

El segundo tipo de accountability educativo es el profesional y apunta a que la escuela y el docente se responsabilicen por brindar experiencias de aprendizajes que respondan a las expectativas y brinden oportunidades a los diferentes estudiantes con los que se trabaja.

Finalmente, el tercer tipo de accountability en sistemas educativos ha sido llamado performativo - por su orientación hacia resultados - o de mercado. Está vinculado con la capacidad de elección de los centros educativos y aporta a que en ese contexto las escuelas compitan por brindar mejor servicio y resultados. Para ello, este mecanismo genera además información, y evaluaciones sobre el logro de las escuelas, además de incentivos que aseguren el cumplimiento de las expectativas definidas para las escuelas.

Educação \& Realidade, Porto Alegre, v. 40, n. 1, p. 275-298, jan./mar. 2015. 


\section{Componentes del Accountability en Sistemas Educativos}

Claramente, el accountability perfomativo es el tipo que cuenta con mayor información o literatura disponible sobre sus componentes ${ }^{1}$. De acuerdo a McMeekin (2006) en educación este mecanismo cuenta con seis elementos:

1. Estándares, que corresponden a expectativas de aprendizaje que describen el desempeño esperado para los estudiantes, docentes, directores o escuelas.

2. Información, se debe producir evaluación que permita conocer si se están alcanzando las expectativas educativas definidas por el sistema. Para ello, en el mundo educativo se utilizan diversos mecanismos, siendo los test estandarizados los más frecuentes.

3. Consecuencias, que apuntan a beneficios o sanciones asociadas al logro o no logro de los estándares.

4. Autoridad, es decir, brindar mayor capacidad a las escuelas para influir en el desarrollo de sus propios procesos, como requisito para poder ser responsabilizados por sus resultados.

5. Desarrollo de capacidades, o brindar las posibilidades para que los que participan de los procesos educativos puedan mejorar sus competencias para cumplir con sus responsabilidades.

6. Comunicación clara y efectiva sobre los objetivos y beneficios del accountability, lo que corresponde a que el entorno valore la utilidad de esta estrategia.

\section{Actores Participantes del Accountability en Sistemas Educativos}

De acuerdo a McMeekin (2006) y Di Gropello (2004), es posible identificar cuatro grandes actores que participan del proceso de accountability y tres grandes relaciones de interacción entre ellos. En relación a los actores, participan 1) los ciudadanos o usuarios que reciben el servicio educacional; 2) políticos y diseñadores de política, que son los que definen objetivos y acciones de mejoramiento desde el estado; 3) proveedores educacionales, que participan en la implementación del servicio educativo (administradores y escuelas); 4) proveedores directos del servicio educacional, es decir, los profesionales de la educación, especialmente los docentes.

Entre estos cuatro actores se observan tres grandes tipos de relaciones. La primera vincula a proveedores (escuelas) y usuarios (estudiantes y apoderados) y es conocida como la ruta corta (DiGropello, 2004). En ella, las familias exigen directamente a las escuelas para que cumplan sus objetivos.

La segunda relación (ruta larga) se establece entre los usuarios y los actores del mundo político y opera por medio de la presión de los 
ciudadanos hacia los políticos, para que estos establezcan mejoras de funcionamiento y de resultados.

Por último, la relación entre el gobierno y los proveedores de servicio (la compacta), consiste en que los organismos públicos asignan recursos, definen objetivos, controlan y actúan para que los proveedores mejoren la entrega de sus servicios.

De lo anterior, se observa que dentro de este modelo la función de los ciudadanos está mayormente asociada a una condición de consumidor del servicio educacional, y como tal su labor corresponde a ejercer presión en base a su elección y preferencias para motivar el mejoramiento de la escuela.

Por su parte, la función del sistema político corresponde a la generación de condiciones, incentivos y normativa que favorezcan el aseguramiento de la calidad en las escuelas. Para ello, supervisan además la labor de las escuelas.

Las escuelas son las principales receptoras de la presión de la ciudadanía y el sistema político, además de la que recibe de su propio administrador. Ellas deben organizarse para responder con los estándares que estos actores ponen a su trabajo. Finalmente, los docentes operan tomando decisiones que favorezcan el cumplimiento de las obligaciones que asumen las escuelas.

\section{El Accountability y su Implementación en Sistemas Educativos en el Mundo, como Mecanismo para Promover el Mejoramiento Educativo}

Los tipos de accountability descritos, sus componentes, los actores involucrados y las relaciones entre estos se han implementado de distintas formas en sistemas educativos en el mundo y a lo largo del tiempo. Claramente el accountability performativo, o de mercado, ha tenido mayor presencia pública y ha sido objeto de múltiples análisis. Sin embargo, incluso dentro de esta tipología, hay disimilitudes en su foco y en su relevancia en los sistemas que lo han implementado. Por esta razón, es relevante realizar un análisis comparado sobre las características que el accountability ha adquirido (y las que no ha asumido) en distintos países, para reconocer de qué manera estos sistemas han incorporado los elementos de este mecanismo en sus estrategias para propiciar el mejoramiento educativo.

Teniendo en cuenta este desafío, a continuación se describirá la manera en que siete sistemas educativos en el mundo han incorporado - o desechado - la opción del accountability, como mecanismo que busca promover el mejoramiento educativo. Para ello, se usarán como referentes los componentes, involucrados y relaciones anteriormente mencionados, poniendo especial énfasis en el diseño del accountability perfomativo y su comparación con opciones de política que prioricen

Educação \& Realidade, Porto Alegre, v. 40, n. 1, p. 275-298, jan./mar. 2015. 279 
tanto las versiones profesional, como la burocrática. Esta separación se hace comprendiendo que en el primer caso se alude a una estrategia educativa con mayor participación del mercado, y en el segundo, con mayor énfasis en la acción de "lo público" en educación.

\section{Análisis de Casos de Sistemas de Accountabilty en Sistemas Educativos en el Mundo}

\section{La Estrategia de Ontario ${ }^{2}$}

Ontario es un estado de Canadá que a partir de fines de la década de los 90 optó por cambiar estrategias orientadas hacia ejercer presión sobre los docentes - con medidas relacionadas con las Reformas Basadas en Estándares ${ }^{3}$-, debido a su alta conflictividad y un desmejoramiento de las condiciones de trabajo de estos actores. Luego, en 2003 se profundizaron los cambios en la estrategia, definiendo políticas focalizadas en el apoyo al desarrollo de capacidades dentro de la escuela, para mejorar el proceso de enseñanza-aprendizaje.

En cuanto a la estrategia de accountability, Ontario cuenta con pruebas para todos los estudiantes en matemáticas e idioma (Inglés o Francés) en los grados 3 y 6, así como de matemáticas en el grado 9. No existen exámenes provinciales de egreso de la escuela.

Las mediciones externas fueron muy controversiales, puesto que se asocian con las políticas de los años 90 en este estado. Sin embargo, de acuerdo a EQAO (2012) esta resistencia habría disminuido, pese a que siguen siendo censales. Junto con ello, se ha buscado desincentivar la preparación de las pruebas y aumentar el uso de la información provista por su aplicación. Además, los test se simplificaron para tomar menos tiempo y el Ministerio desalienta las comparaciones entre escuelas (2012).

Junto con los test, Ontario motiva a la provincia, los distritos escolares y escuelas a que informen públicamente sobre una variedad de indicadores de progreso del estudiante.

De manera complementaria, se ha implementado la ley "Poner a los estudiantes primero", que en su discurso reconoce que los docentes necesitan flexibilidad suficiente para tomar decisiones, pero se asume también que la administración del sistema debe proveer una lista de instrumentos de evaluación previamente aprobados desarrollados por la junta escolar local, que sean coherentes con planes de mejora.

\section{El Caso Colombiano ${ }^{4}$}

En el caso colombiano, la lógica del sistema de accountability se vincula con las definiciones de su Constitución, la que define acciones de deben ser abordadas a nivel local. El decreto 1290 del año 2009 regla- 
menta la evaluación del aprendizaje y promoción de los estudiantes que deben realizar los establecimientos educativos. Ahí se indica explícitamente que las evaluaciones son un proceso eminentemente interno. Con ello, se busca declarar confianza en el cumplimiento de esta función de parte de los docentes, así como la promoción de las definiciones evaluativas contextualizadas con las realidades educativas y características de los estudiantes (Ministerio de Educación Nacional, 2009).

La Ley General de Educación de ese país (artículo 77) otorga la autonomía escolar a las instituciones en cuanto a los siguientes elementos: organización de las áreas fundamentales, inclusión de asignaturas optativas, ajuste del proyecto educativo institucional (PEI) a las necesidades y características regionales, libertad para la adopción de métodos de enseñanza y la organización de actividades formativas, culturales y deportivas. Bajo esta ley, y considerando el decreto 1290, se organiza un Sistema Institucional de Evaluación, que define de escalas de valoración o desempeños esperados para la institución educativa, como parámetro general común a las distintas instituciones educativas.

Cada escuela debe construir informes de evaluación, así como declarar su periodicidad. En cuanto a la publicación de los resultados, los padres de familia deben tener acceso permanente a la información correspondiente de la evaluación de los aprendizajes de sus hijos.

Respecto de las responsabilidades del Ministerio de Educación Nacional (MEN), aparte de definir una escala de valoración nacional (niveles de logro esperados para la evaluación), el MEN define lineamientos y establece las normas técnicas curriculares y pedagógicas. En esa lógica, ha publicado Lineamientos Curriculares, Estándares Básicos de Competencias y Orientaciones Pedagógicas o Generales, para las áreas fundamentales ${ }^{5}$.

Desde las políticas educativas, se han orientado otras acciones hacia el aseguramiento de la calidad de la educación, tales como Planes de Mejoramiento Educativo, tanto para las escuelas, como para las secretarías de educación regionales. A ello se suman sistemas de incentivos o estímulos y acciones de inspección o vigilancia. Por su parte, desde el punto de vista de la medición externa, se incorporan las pruebas SABER. Estas miden el logro de los estándares educativos y se implementan en el tercer, quinto y noveno año de enseñanza, fundamentalmente en lenguaje y matemática y en algunos años, ciencias naturales y competencias ciudadanas. Hasta la fecha, los resultados de las pruebas SABER no están asociados a consecuencias.

\section{El Caso de Estados Unidos y la Ley No Child Left Behind ${ }^{6}$}

El caso estadounidense se organiza desde el nivel federal, a través de la Ley No Child Left Behind (NCLB) y ha sido ampliamente debatido y muy controversial. Una de sus principales características es la 
Accountabilty y Mejoramiento Educativo

obligación de mostrar públicamente los resultados académicos de las escuelas y responsabilizarse por ellos. Cada estado debe medir anualmente el progreso de cada alumno de las escuelas públicas en lectura, matemática y ciencia, desde el tercer hasta el octavo grado y al menos una vez durante los grados 10 al 12. Estas evaluaciones deben ajustarse al contenido y los objetivos mínimos de aprendizaje establecidos por cada estado. Además de las pruebas estatales, los estudiantes de cuarto y octavo grado deben rendir una medición de carácter federal, denominada NAEP, para complementar información y favorecer la comparación entre estados.

La ley prioriza que la información generada en estas pruebas esté dirigida hacia los padres, para favorecer la elección de escuelas, como mecanismo de presión orientado hacia el mejoramiento. Respecto de su contenido, deben indicar cuántos estudiantes de cada escuela alcanzaron niveles básicos, aceptables y avanzados de resultados. Además, se señala si las escuelas necesitan acciones correctivas o cambios mayores.

A través de NCLB las escuelas reciben fondos federales especiales, que deben usarse para desarrollar procesos de mejoramiento.

Si una escuela logra persistentemente malos resultados, la ley indica que sus alumnos podrían ser trasladados escuelas de rendimiento más alto en la misma zona o prestarles servicios educativos suplementarios en la comunidad, como tutorías o clases de recuperación.

NCLB mandata a que cada estado defina el "Progreso Anual Adecuado" (AYP) para los distritos escolares y las escuelas, dentro de los parámetros fijados por la Ley Federal.

En relación las consecuencias asociadas al mecanismo, la Ley indica que en caso de no lograr el AYP, las escuelas van afectando su categoría y con ello sus posibilidades de operación. Al tercer año sin obtener mejoras en resultados, el estado debe ofrecer a los estudiantes la opción de trasladarse a otra escuela pública del distrito. Al cuarto año, el distrito deberá tomar acciones correctivas orientadas al mejoramiento de la escuela. Finalmente, al quinto año de no mejoramiento en resultados, el distrito escolar debe iniciar planes de reestructuración del establecimiento.

\section{El Caso Finés ${ }^{7}$}

Desde hace décadas que Finlandia cuenta con objetivos declarados de proporcionar a todos los estudiantes las mismas oportunidades educativas. Su sistema educativo se caracteriza por su no selectividad, por la relevancia de su educación pública y la obligación de asegurar calidad para todos. En este marco, es el profesor quien asume la obligación de adaptarse a las necesidades de los diferentes niños que participan de la escuela, así como la imposibilidad de excluir o derivar alumnos 
a otras unidades educativas. El docente es considerado idiosincráticamente como un experto en su trabajo, siendo altamente valorado por la sociedad. Por ello, se delega autonomía a las escuelas para identificar las mejores metodologías para enseñar a sus estudiantes.

La política educativa finesa se caracteriza por el uso explícito de conceptos como el de confianza, respeto por la diversidad, por el trabajo de los profesores y la relevancia de los niveles locales (Dirección Nacional de Educación, 2007; Ley de Educación, 1998). De esta forma, la responsabilización del profesor en relación a su propio trabajo es parte constituyente de su rol. El estado, por su parte, asume la necesidad de invertir en sus profesores, por medio de su formación continua.

El principio de la equidad es el otro elemento de base en la política educativa finesa y su objetivo es que las distintas escuelas provean de un tipo y calidad de educación similar.

Las mediciones externas de aprendizaje tienen un carácter solo muestral y generan información pública hacia el sistema, pero lo que sucede con cada escuela se entrega directamente a su comunidad.

El término accountability no existe en el sistema educativo finés, pese al nivel de descentralización de las decisiones educativas en las escuelas y los docentes. No se fomentan tampoco políticas asociadas a altas consecuencias, y se espera proteger a las escuelas de tipos de competencias como las que se alientan en el accountability performativo.

\section{El Caso de Singapur ${ }^{8}$}

En 1992, Singapur empezó a clasificar anualmente a las escuelas y a publicar sus resultados en los medios de comunicación locales. A partir de entonces, el sistema ha ido adoptando mecanismos que progresivamente variaron el foco de las expectativas de evaluación y mejoramiento educativo. En el año 2000, se incorporó la autoevaluación de las escuelas como mecanismo de evaluación, considerando un llamado Modelo de Excelencia Escolar (SEM). En este modelo se consideran resultados académicos (exámenes a nivel nacional y otras áreas clave de aprendizaje), pero se requiere del análisis de otras dimensiones para completar la autoevaluación. Cada escuela tiene que utilizar el SEM para llevar a cabo la autoevaluación anual. Junto con ello, un equipo externo del Ministerio de Educación valida los resultados de la autoevaluación aproximadamente una vez en 5 años.

En paralelo, fue adquiriendo relevancia un movimiento denominado "Enseñar menos y aprender más", orientando hacia el autoaprendizaje y el desarrollo de procesos de aprendizaje profundos, buscando mejorar la calidad de la educación.

$\mathrm{Ng}$ (2010) señala que este país concluyó que en los 90 existía un exceso de confianza en las evaluaciones externas y en su poder de propiciar el mejoramiento. Por ello, se dio un giro haca un concepto de cali-

Educação \& Realidade, Porto Alegre, v. 40, n. 1, p. 275-298, jan./mar. 2015. 283 
Accountabilty y Mejoramiento Educativo

dad educativa que se sostiene en el desarrollo de competencias internas de parte de las escuelas.

Pese a la relevancia de la autoevaluación, el sistema de Singapur es especialmente centralizado en cuanto a su administración. Ng (2012) señala que, aunque se afirme oficialmente que la evaluación y la información está dirigida hacia los apoderados y la comunidad, en realidad prevalece el accountability hacia el Estado. Las escuelas son públicas y los docentes son funcionarios públicos, lo que ha incidido en que se responda mayoritariamente al Ministerio de Educación, en vez que a juntas escolares. Tampoco existe la dependencia financiera de los apoderados, puesto que el presupuesto de las escuelas es público. Además, las organizaciones comunitarias no tienen gran injerencia en la toma de decisiones.

Finalmente, es importante destacar la gran inversión que hace este país en el desarrollo profesional docente y directivo. Los docentes y directores reciben una alta remuneración y, para ello, deben responder exitosamente a fuertes procesos de selección en el caso de la formación inicial, así como de formación continua.

Respecto de los objetivos educativos esperados por el sistema, El Ministerio de Educación singapurense indica al respecto que los "resultados deseados en educación" (DOE) establecen un propósito común para los educadores y entregan una orientación para las políticas educativas y permiten determinar una medida de calidad del sistema. Estas expectativas definidas para los estudiantes son más amplias que las competencias de carácter cognitivo y apuntan a diferentes dimensiones de la vida personal y social.

\section{El Caso Inglés ${ }^{9}$}

En este país se asumió tempranamente la estrategia de Reforma Basada en Estándares e incorporó el concepto de accountability, aunque esta perspectiva ha ido modificándose en el tiempo. El Ministerio de Educación y las oficinas responsables indican explícitamente la relevancia del término accountability, definido en la Estrategia de Escuelas Primarias y el Plan de Escuelas Públicas.

En el centro del modelo de mejoramiento del sistema educativo inglés se señala que están los estudiantes y sus logros esperados y se definen tres grandes elementos para alcanzarlo: el plan de mejoramiento educativo, la evaluación de sus logros y el mejoramiento de ese plan.

Los procesos de evaluación se asumen como hitos internos y externos para verificar el cumplimiento del desempeño escolar. Ambos tipos de evaluación son obligatorios. El núcleo de las evaluaciones son los estándares y se indica explícitamente que las escuelas son las responsables de alcanzarlos. La escuela está obligada a: a) implementar una autoevaluación, realizada con los estándares aprendizaje escolar 
y de eficacia de los procesos escolares; b) emprender procesos de planificación escolar; c) publicar anualmente un Informe Escolar sobre el desempeño de la escuela y otro sobre cumplimiento de los requisitos de las políticas educativas (sobre éste se hace una clasificación de las escuelas); y d) promover la participación en los procesos de revisión de la escuela.

Las escuelas rinden cuenta de su trabajo ante la autoridad local, respecto del uso de recursos y a Ofsted - entidad gubernamental a cargo de los estándares y la evaluación - respecto de sus logros académicos. Los resultados de estas evaluaciones están asociados a consecuencias. Además, la Ofsted realiza visitas de inspección a las escuelas, cuyos informes afectan la categoría del establecimiento.

La evaluación y clasificación de escuelas se presenta como un insumo que favorece la elección de escuelas, y con ello su competencia. Además, se ha legislado para poner a disposición una gran cantidad de información para que los consumidores puedan exigir resultados y elegir.

\section{El Caso Chileno}

El estado chileno ha generado continuamente sistemas y mecanismos con los que espera avanzar paralelamente en los desafíos de calidad y equidad en la educación (Bellei; Contreras; Valenzuela, 2010; Martinic, 2010). Uno de los más relevantes es la consolidación del uso de la evaluación sobre el desempeño académico de los estudiantes, como mecanismo que favorece el mejoramiento de la acción y resultados. Paulatinamente, la medición nacional de resultados de aprendizaje (SIMCE) se ha convertido en un eje para la rendición de cuentas de las escuelas, su ordenación respecto de su desempeño y la vinculación de ello con incentivos, como mecanismo movilizador del mejoramiento (Weinstein; Muñoz, 2012).

En Chile, las iniciativas asociadas con esta lógica surgen hace cerca de 15 años. Sin embargo, con la promulgación de la Ley de Subvención Escolar Preferencial en 2008, la función de la evaluación se consolida como mecanismo movilizador de la expectativa de mejoramiento en el sistema educativo, a través de instalación de dispositivos de consecuencias asociadas al desempeño. Para promover el mejoramiento, se proporcionan recursos financieros, asociados a la implementación de un Plan de Mejoramiento Educativo a 4 años. Para apoyar este proceso, se creó un cuasimercado de asistencia técnica educativa privada.

La política recién descrita se consolida el año 2012, con la Ley 20.529, que define un Sistema Nacional de Aseguramiento de la Calidad de la Educación y estructura un funcionamiento organizado y sistémico de los componentes de las Reformas Basadas en Estándares. En ella, se consolida la función del estado de ordenar a las escuelas en base a

Educação \& Realidade, Porto Alegre, v. 40, n. 1, p. 275-298, jan./mar. 2015. 285

Disponível em: <http://www.ufrgs.br/edu_realidade> 
Accountabilty y Mejoramiento Educativo

su desempeño y se define que el factor prioritario de determinación de calidad educativa es el SIMCE.

En este período se pusieron en marcha dos nuevas instituciones estatales con responsabilidad educativa. La Agencia de Calidad de la Educación, debe implementar la evaluación, inspeccionar escuelas, ordenarlas en base al desempeño e informar a la comunidad. La Superintendencia de Educación, vela por el cumplimiento de la normativa de parte de las escuelas y del organismo que las administra.

La información sobre el desempeño de la escuela, el cumplimiento de la normativa y el uso de los recursos son insumos que la institucionalidad educativa comunica a la ciudadanía para la elección de las escuelas. Asimismo, la legislación incluye consecuencias asociadas al logro de los resultados educativos, como la disminución en la autonomía en el uso de los recursos, o incluso la pérdida del reconocimiento oficial de la escuela. El estado debe asegurar la matrícula de los estudiantes de las escuelas que deban ser cerradas en función de sus bajos resultados.

\section{Análisis Comparado de los Casos deAccountability Descritos}

A continuación se describe un análisis de la información descrita sobre los casos y su uso del accountability como mecanismo para favorecer el mejoramiento educativo. Para ello, se utilizarán como criterios los componentes de este mecanismo y el rol de los actores involucrados, y se evaluará su presencia o relevancia para cada sistema educativo.

Considerando los conceptos que se trabajaron respecto de esta estrategia, se pondrá especial énfasis en el establecimiento de comparaciones entre las opciones de política más cercanas al accountability performativo, o de mercado, respecto de otras opciones posibles, tales como la versión profesional y burocrática del accountability. Esta estructura dicotómica en el análisis se mantendrá en las conclusiones del trabajo, dado que incorporan el principal debate entre las opciones de uso y la valoración de este mecanismo: una orientada hacia el mercado y otra mayormente dominada por el estado y por el concepto de lo público.

Respecto de la presencia de estándares de aprendizaje en los sistemas analizados (ver Figura 1), se observan elementos que no permiten discriminar completamente entre las estrategias asumidas por cada caso. Existen países caracterizados por diseños de accountability performativo que cuentan con estándares desde los que realizan todo el proceso evaluativo de aprendizajes y de eficacia de las escuelas. Sin embargo, Colombia también cuenta con estándares, pero no es el gobierno central quien los aplica en las evaluaciones vinculadas a los procesos de accountability, sino que se utilizan de manera descentralizada.

En relación con la información que genera el sistema, tanto para la rendición de cuentas, como para la responsabilización de los actores 
educativos, sí se observan perfiles más claros y diferenciados entre los casos. Mientras Colombia y Finlandia relevan el valor de la evaluación interna, en el otro extremo hay países que sostienen su accountability desde la evaluación externa. Esto ocurre con los casos estadounidense y chileno. Finalmente, al medio de estos dos grupos se ubican Singapur, Inglaterra y Ontario, que combinan ambos recursos de información en la generación de la evaluación que realizan sobre las escuelas. Estos tres casos utilizan información levantada centralmente e incluyen de manera institucionalizada la información provista por el nivel local en el proceso de accountability.

Las consecuencias asociadas a los mecanismos de accountability permiten agrupar nuevamente a un conjunto de países con un perfil más claramente performativo (Chile y Estados Unidos), que incluyen altas consecuencias, asociadas al cumplimiento de los estándares y expectativas de logro. A ellos se suma Inglaterra, donde las consecuencias se encuentran incorporadas en su marco normativo.

En los otros casos hay patrones menos claros de presencia de consecuencias. Por ejemplo, en Ontario y Singapur se asumen consecuencias asociadas a la evaluación, pero con plazos más amplios u otro tipo decisiones asociadas. Por su parte, el caso finés no incluye consecuencias formalmente. Sin embargo, sí se asume un alto nivel de responsabilización interna de los actores educativos en el proceso, asociada a la confianza en el sistema.

Finalmente, Colombia no asume consecuencias vinculadas al proceso de rendición de cuentas de los resultados educativos de las escuelas.

En relación a la autoridad, o capacidad de agencia a nivel local, los países del polo performativo se caracterizan por un bajo nivel de autoridad, debido a la relevancia de los estándares que son definidas a nivel central. Debido a la relación existente con el poder del nivel central en esta materia, a este grupo se suma en esta ocasión el caso de Singapur, por el amplio poder que tiene el proyecto nacional de educación en la toma de decisiones locales. Los otros casos muestran progresivamente mayores niveles de autoridad a nivel local. Inglaterra cuenta con un nivel intermedio, debido a que el espacio local tiene margen para hacer definiciones propias de expectativas de logro, las que deben convivir con el poder centralizado de los estándares y la evaluación. Finalmente, los casos de Ontario, Colombia y Finlandia se caracterizan por mayores grados de autonomía local en la toma de decisiones. En ese sentido, Colombia y Finlandia hacen mayormente explícito este principio de autonomía, en términos de las definiciones de los parámetros para evaluar el cumplimiento de los objetivos educativos.

Respecto del rol del desarrollo de capacidades en los sistemas educativos, se observan grupos más homogéneos, pues en la mayoría de estos países se hace explícito este esfuerzo, lo que se observa más clara-

Educação \& Realidade, Porto Alegre, v. 40, n. 1, p. 275-298, jan./mar. 2015. 287 
mente en los casos de Ontario, Singapur, Finlandia, junto con Inglaterra, Estados Unidos y Chile, en un menor grado que el resto. El ejemplo de Singapur es especialmente llamativo en esta materia. Por su parte, en el caso colombiano este esfuerzo es menos evidente en el discurso y en las disposiciones normativas.

Finalmente, en cuanto a la instalación de una cultura de accountability, vuelven a existir importantes diferencias entre las definiciones de los casos analizados. Al respecto, nuevamente vuelve a configurarse el polo asociados al accountability performativo (Estados Unidos, Chile e Inglaterra), que promueven una cultura exteriorizada, orientada hacia la elección de escuelas en base a sus resultados. Este grupo contrasta con el finés, donde la cultura de la responsabilización es alta, pero está internalizada en los actores educativos. Por su parte, los casos de análisis de Ontario y Singapur, cuentan con definiciones intermedias, porque tanto la evaluación como sus efectos se nutren con insumos externos e internos a la escuela, lo que vincula a estos modelos más con el accountability de tipo profesional. Finalmente, en el caso colombiano están menos presentes los discursos referidos al accountability (exteriorizado o internalizado), en la cultura de mejoramiento del sistema educativo.

\section{Cuadro 1 - Características del Accountability en los Sistemas} Educativos Analizados

\begin{tabular}{|l|l|l|l|l|l|l|}
\hline Caso & $\begin{array}{l}\text { Estánda- } \\
\text { res }\end{array}$ & $\begin{array}{l}\text { Infor- } \\
\text { mación }\end{array}$ & $\begin{array}{l}\text { Conse- } \\
\text { cuencias }\end{array}$ & $\begin{array}{l}\text { Autori- } \\
\text { dad }\end{array}$ & $\begin{array}{l}\text { Desar- } \\
\text { rollo de } \\
\text { capaci- } \\
\text { dades }\end{array}$ & $\begin{array}{l}\text { Cultu- } \\
\text { ra de } \\
\text { accoun- } \\
\text { tability }\end{array}$ \\
\hline Ontario & Sí & $\begin{array}{l}\text { Evalu- } \\
\text { ación } \\
\text { interna y } \\
\text { externa } \\
\text { (censal) }\end{array}$ & Bajas & Alta & Alta & $\begin{array}{l}\text { Como } \\
\text { recurso } \\
\text { de apoyo }\end{array}$ \\
\hline Colombia & Sí & $\begin{array}{l}\text { Infor- } \\
\text { mación } \\
\text { predo- } \\
\text { minan- } \\
\text { temente } \\
\text { interna. }\end{array}$ & No & Alta & Baja & $\begin{array}{l}\text { No } \\
\text { desar- } \\
\text { rollado }\end{array}$ \\
\hline $\begin{array}{l}\text { Estados } \\
\text { Unidos }\end{array}$ & Sí & $\begin{array}{l}\text { Predo- } \\
\text { minio } \\
\text { evalu- } \\
\text { ación } \\
\text { externa }\end{array}$ & Altas & Baja & $\begin{array}{l}\text { Interme- } \\
\text { dia }\end{array}$ & $\begin{array}{l}\text { Respon- } \\
\text { sabili- } \\
\text { zación } \\
\text { externa }\end{array}$ \\
\hline $\begin{array}{l}\text { Finlan- } \\
\text { dia }\end{array}$ & No & $\begin{array}{l}\text { Predo- } \\
\text { minio } \\
\text { evalu- } \\
\text { ación } \\
\text { interna }\end{array}$ & $\begin{array}{l}\text { Bajas de } \\
\text { carácter } \\
\text { externo }\end{array}$ & Alta & Alta & $\begin{array}{l}\text { Respon- } \\
\text { sabili- } \\
\text { zación } \\
\text { Interna }\end{array}$ \\
\hline
\end{tabular}




\begin{tabular}{|l|l|l|l|l|l|l|}
\hline Singapur & $\begin{array}{l}\text { Sí, pero a } \\
\text { nivel de } \\
\text { expecta- } \\
\text { tivas de } \\
\text { funcio- } \\
\text { namien- } \\
\text { to de } \\
\text { escuela }\end{array}$ & $\begin{array}{l}\text { Evalu- } \\
\text { ación } \\
\text { interna y } \\
\text { externa }\end{array}$ & Bajas & Baja & Alta & $\begin{array}{l}\text { Como } \\
\text { recurso } \\
\text { de apoyo }\end{array}$ \\
\hline $\begin{array}{l}\text { Ingla- } \\
\text { terra }\end{array}$ & Sí & $\begin{array}{l}\text { Evalu- } \\
\text { ación } \\
\text { interna y } \\
\text { externa }\end{array}$ & Altas & $\begin{array}{l}\text { Interme- } \\
\text { dia }\end{array}$ & $\begin{array}{l}\text { Interme- } \\
\text { dia }\end{array}$ & $\begin{array}{l}\text { Respon- } \\
\text { sabili- } \\
\text { zación } \\
\text { externa }\end{array}$ \\
\hline Chile & Sí & $\begin{array}{l}\text { Predo- } \\
\text { minio } \\
\text { evalu- } \\
\text { ación } \\
\text { externa }\end{array}$ & Altas & Baja & Baja & $\begin{array}{l}\text { Respon- } \\
\text { sabili- } \\
\text { zación } \\
\text { externa }\end{array}$ \\
\hline
\end{tabular}

\section{El Rol de los Actores Educativos en la Estructura del Accountability}

El análisis de la estructura del accountability en los casos analizados se completa con la revisión del rol de los principales actores vinculados a este proceso: ciudadanía, gobierno, escuelas y docentes.

En el caso de la ciudadanía, el polo de accountability perfomativo considera un rol de consumidor de servicios educacionales. Los padres y apoderados, y la comunidad en general, tomarían decisiones respecto de dónde educar a sus hijos, basados en la información que proporcione el sistema sobre el desempeño de las escuelas. Por su parte, casos como el de Ontario y Singapur definen una posición intermedia para la ciudadanía, a quien se informa sobre los resultados de las escuelas, pero que además debe asumir un rol de comunidad educativa. Por su parte, los casos de Colombia y Finlandia se alejan en mayor medida de la condición de ciudadano como consumidor, proporcionando un mayor peso político al rol de la ciudadanía. En Finlandia se estima que la ciudadanía es garante de la autonomía escolar, como condición para el trabajo profesional de las escuelas. En Colombia, la ciudadanía se encuentra próxima a la toma de decisiones respecto de definiciones clave de expectativas u objetivos educativos.

Por su parte, en relación al rol del gobierno central en este mecanismo, es llamativo que en este caso una mayor parte de los casos se caracterice por un alto control de la burocracia estatal respecto de los procesos y resultados educativos. En esta dimensión hay menos diferencias entre los países, y la función que es prácticamente transversal a los distintos casos analizados es la evaluación. Los casos que se escapan en mayor medida de esta característica son, nuevamente, Colombia y Finlandia. Respecto del primer país, la medición externa

Educação \& Realidade, Porto Alegre, v. 40, n. 1, p. 275-298, jan./mar. 2015. 289

Disponível em: <http://www.ufrgs.br/edu_realidade> 
Accountabilty y Mejoramiento Educativo

es censal, pero no está vinculada con consecuencias, o decisiones de financiamiento de parte del gobierno central. En el caso de Finlandia, las pruebas son muestrales y se declara explícitamente que no existirán dichas consecuencias.

Los casos vuelven a diferenciarse respecto del rol definido para las escuelas, puesto que los países del polo performativo esperan un funcionamiento menos autónomo de las escuelas, para que puedan ajustar su funcionamiento y cumplir con los objetivos nacionales o estatales. Pese a ello, allí las escuelas son consideradas como las principales responsables de éxito o fracaso educativo. A este grupo se suma el caso de Singapur, debido al rol mayormente ejecutor de las políticas educativas estatales. Sin embargo, este país se diferencia del polo performativo en las características y contenido de la evaluación y respecto de la responsabilidad de las escuelas. Por su parte, Ontario nuevamente muestra una organización mixta, puesto que ponen énfasis en la responsabilidad de las escuelas, pero se asume un mayor protagonismo de ellas en la definición de expectativas y prioridades educativas. Esta última condición es también compartida por los casos colombiano y finés. Este último país mantiene una gran relevancia de la responsabilidad de las escuelas, pero esta tiene una determinación más interna que externa.

La estructura recién descrita se replica en el caso de los docentes. En este caso, parece ser relevante destacar que los casos de Ontario y especialmente el de Finlandia son los que el docente muestra mayor protagonismo, en cuanto a la autonomía que dispone para el diseño de su trabajo y la definición de prioridades para la enseñanza.

\section{Cuadro 2 - Rol de los Actores Participantes en el Sistema}

\begin{tabular}{|c|c|c|c|c|}
\hline Casso & Ciudadanos & Gobierno & Escuelas & Docentes \\
\hline Ontario & $\begin{array}{l}\text { Reciben infor- } \\
\text { mación para } \\
\text { cumplir un rol } \\
\text { en proceso de } \\
\text { mejoramiento } \\
\text { y se informa } \\
\text { con pruebas } \\
\text { externas }\end{array}$ & $\begin{array}{l}\text { Define } \\
\text { estándares, } \\
\text { promueve me- } \\
\text { joramiento y } \\
\text { apoya. Evalúa }\end{array}$ & $\begin{array}{l}\text { Definen pro- } \\
\text { cesos de mejo- } \\
\text { ramiento. Son } \\
\text { responsables, } \\
\text { como otros } \\
\text { organismos }\end{array}$ & $\begin{array}{l}\text { Toman de- } \\
\text { cisiones de } \\
\text { mejoramien- } \\
\text { to. Reciben } \\
\text { información } \\
\text { y apoyo y se } \\
\text { espera que } \\
\text { cumplan los } \\
\text { objetivos que } \\
\text { declaran }\end{array}$ \\
\hline Colombia & $\begin{array}{l}\text { Comunidad } \\
\text { participa en } \\
\text { definiciones } \\
\text { de evaluación } \\
\text { y se informa } \\
\text { con pruebas } \\
\text { externas }\end{array}$ & $\begin{array}{l}\text { Define } \\
\text { estándares. } \\
\text { Promueve } \\
\text { evaluación } \\
\text { local. Evalúa } \\
\text { sin conse- } \\
\text { cuencias }\end{array}$ & $\begin{array}{l}\text { Definen } \\
\text { evaluaci- } \\
\text { ón. Toman } \\
\text { decisiones de } \\
\text { mejoramien- } \\
\text { to. Se respon- } \\
\text { sabilizan por } \\
\text { cumplimiento } \\
\text { de normativa }\end{array}$ & $\begin{array}{l}\text { Enseñan y } \\
\text { evalúan bajo } \\
\text { definicio- } \\
\text { nes locales } \\
\text { y normativa } \\
\text { nacional }\end{array}$ \\
\hline
\end{tabular}




\begin{tabular}{|c|c|c|c|c|}
\hline $\begin{array}{l}\text { Estados } \\
\text { Unidos }\end{array}$ & $\begin{array}{l}\text { Eligen oferta } \\
\text { educativa } \\
\text { y supervisa } \\
\text { desempeño } \\
\text { de escuelas } \\
\text { en base a in- } \\
\text { formación de } \\
\text { evaluación. }\end{array}$ & $\begin{array}{l}\text { Define } \\
\text { estándares, fi- } \\
\text { nancia, evalúa } \\
\text { con conse- } \\
\text { cuencias. } \\
\text { Informa sobre } \\
\text { resultados }\end{array}$ & $\begin{array}{l}\text { Son respon- } \\
\text { sables de los } \\
\text { resultados } \\
\text { (externos) }\end{array}$ & $\begin{array}{l}\text { Enseñan el } \\
\text { currículum y } \\
\text { buscan alcan- } \\
\text { zar estándares }\end{array}$ \\
\hline Finlandia & $\begin{array}{l}\text { Espera igual- } \\
\text { dad y calidad } \\
\text { educativa y } \\
\text { participa a } \\
\text { nivel local }\end{array}$ & $\begin{array}{l}\text { Define expec- } \\
\text { tativas para } \\
\text { el sistema. } \\
\text { Financia } \\
\text { desarrollo de } \\
\text { capacidades. } \\
\text { Monitorea } \\
\text { funciona- } \\
\text { miento del } \\
\text { sistema }\end{array}$ & $\begin{array}{l}\text { Son respon- } \\
\text { sables de } \\
\text { cumplimiento } \\
\text { de objetivos } \\
\text { (interna) }\end{array}$ & $\begin{array}{l}\text { Son protago- } \\
\text { nistas del sis- } \\
\text { tema. Toman } \\
\text { decisiones y } \\
\text { tienen amplia } \\
\text { autonomía }\end{array}$ \\
\hline Singapur & $\begin{array}{l}\text { Participa de } \\
\text { sistema a } \\
\text { nivel local y se } \\
\text { informa con } \\
\text { evaluaciones } \\
\text { internas y } \\
\text { externas }\end{array}$ & $\begin{array}{l}\text { Define ex- } \\
\text { pectativas. } \\
\text { Supervisa } \\
\text { autoevaluaci- } \\
\text { ón. Financia. } \\
\text { Controla fun- } \\
\text { cionamiento } \\
\text { de escuelas }\end{array}$ & $\begin{array}{l}\text { Implementan } \\
\text { políticas y } \\
\text { programas. } \\
\text { Son respon- } \\
\text { sables de } \\
\text { procesos y } \\
\text { resultados, sin } \\
\text { altas conse- } \\
\text { cuencias }\end{array}$ & $\begin{array}{l}\text { Implementan } \\
\text { políticas, pla- } \\
\text { nes y progra- } \\
\text { mas. }\end{array}$ \\
\hline Inglaterra & $\begin{array}{l}\text { Elige oferta } \\
\text { educativa } \\
\text { y supervisa } \\
\text { desempeño } \\
\text { de escuelas } \\
\text { en base a in- } \\
\text { formación de } \\
\text { evaluación }\end{array}$ & $\begin{array}{l}\text { Define están- } \\
\text { dares. Evalúa } \\
\text { con conse- } \\
\text { cuencias. } \\
\text { Informa sobre } \\
\text { resultados } \\
\text { de evaluaci- } \\
\text { ón interna y } \\
\text { externa }\end{array}$ & $\begin{array}{l}\text { Son respon- } \\
\text { sables de } \\
\text { cumplimiento } \\
\text { de resultados } \\
\text { y obligaciones } \\
\text { de modelo } \\
\text { educativo }\end{array}$ & $\begin{array}{l}\text { Enseñan el } \\
\text { currículum y } \\
\text { buscan cum- } \\
\text { plir estánda- } \\
\text { res }\end{array}$ \\
\hline Chile & $\begin{array}{l}\text { Elige oferta } \\
\text { educativa } \\
\text { y supervisa } \\
\text { desempeño } \\
\text { en base a in- } \\
\text { formación de } \\
\text { evaluación }\end{array}$ & $\begin{array}{l}\text { Evalúa con } \\
\text { consecuen- } \\
\text { cias. Informa } \\
\text { sobre resulta- } \\
\text { dos de evalu- } \\
\text { ación externa. } \\
\text { Financia }\end{array}$ & $\begin{array}{l}\text { Son respon- } \\
\text { sables de } \\
\text { resultados } \\
\text { educativos }\end{array}$ & $\begin{array}{l}\text { Enseñan el } \\
\text { currículum y } \\
\text { buscan alcan- } \\
\text { zar aprendi- } \\
\text { zajes que son } \\
\text { ratificados } \\
\text { por medición } \\
\text { externa }\end{array}$ \\
\hline
\end{tabular}

\section{Conclusiones}

De la descripción y análisis de los casos estudiados, ha sido importante observar la variedad y diversidad de opciones y la combinación de estrategias con las que el sistema educativo busca responsabilizarse y rendir cuentas por el cumplimiento de los objetivos y expectativas que existen respecto de la educación, construyendo así un sistema propio de accountability.

Educação \& Realidade, Porto Alegre, v. 40, n. 1, p. 275-298, jan./mar. 2015. 
Accountabilty y Mejoramiento Educativo

El análisis realizado muestra que solo un segmento de la muestra analizada optó por un tipo performativo, o de mercado. Por su parte, el caso finés es el que más se acerca al tipo puro de accountability profesional, aunque es importante constatar que el lenguaje de este sistema no incorpora una adscripción o valoración explícita del término accountability. Asimismo, Singapur es el caso más cercano al modelo burocrático.

Este estudio muestra en algunos casos además la incorporación de elementos provenientes de otras tipologías, por ejemplo, a nivel de medición de aprendizajes. El caso de Ontario es el de mayor sincretismo entre las tipologías de accountability, puesto que pueden verse claramente en sus definiciones elementos orientados a la visión burocrática, profesional y performativa. Finalmente, el caso colombiano también adopta diferentes componentes, propios de distintos modelos.

Considerando esta revisión, se observa que los casos analizados transitan desde un "polo performativo", mayormente basado en la lógica del mercado, hacia un "polo público", relacionado con un mayor protagonismo del estado en vinculación con las comunidades educativas.

De las normas y análisis recogidos no puede decirse que el término accountability sea transversalmente utilizado. Pese a ello, paradójicamente una parte de la bibliografía disponible acoge este concepto como un mecanismo de utilidad prácticamente universal, especialmente cuando la literatura hace mención al accountability perfomativo, o de mercado. Es decir, hay una tendencia hacia la monopolización de una perspectiva respecto del diseño de este mecanismo, siendo que solo un subconjunto de sistemas educativos los que lo han implementado "tal como lo dicta el libro".

Teniendo esto en consideración, ¿̇será adecuado o útil señalar que los casos que no pertenecen al polo performativo también tienen sistemas de accountability? ¿ No será mejor indicar que son otras estrategias de mejoramiento educativo, que no están necesariamente vehiculizadas por ese concepto?

Otro elemento interesante que se constató es que en tres de los casos estudiados, luego de implementar procesos de accountability performativo, se optó por retroceder en esa opción, otorgando mayores facultades al nivel educativo local, para la evaluación y la definición de objetivos. Mientras, Estados Unidos ha persistido con la idea y Chile ha profundizado en esa opción. Por su parte, Finlandia no muestra ningún interés en acercarse a esa visión. ¿Cuáles son las motivaciones para abandonar, profundizar o no considerar esta estrategia? Esta pregunta no puede ser respondida en este trabajo, pero aparece como una temática de relevancia para ser profundizada por la investigación.

En el caso de los que han ido abandonando el accountability performativo, se hace explícita una evaluación sobre su eficacia (no se estaba mejorando en la medida que se esperaba) y sobre su sentido (se con- 
sideró que no era la forma en que se debía mejorar). En paralelo, el caso finés considera que sus objetivos educativos no se alcanzan a través del mecanismo de accountability. En ambas decisiones es posible apreciar como elemento común la valoración de los focos de estudio actual del mejoramiento educativo - descritos al inicio de este trabajo. Es posible interpretar que en estos casos se asume que el accountability performativo no permite alcanzar los desafíos que presenta este proceso.

El análisis recién mencionado favorece la reflexión respecto a qué tan indispensable es la existencia completa de los componentes del accountability y relaciones entre sus actores, para promover el mejoramiento educativo. Por lo que puede analizarse de los casos, sobre todo de aquellos que tienen un presente exitoso en materia de educación, lo que aparece como indispensable es que existan dinámicas de favorezcan la responsabilización de los actores respecto de su trabajo. Sin embargo, no es evidente si este proceso debiera ser producido a partir de presión ejercida desde fuera, o por un sentido de responsabilidad internalizado.

Además, la descripción de los casos también muestra que el mecanismo de accountability no permitiría por sí mismo alcanzar procesos de mejoramiento educativo, ya que será necesario que se generen sistemas igualmente robustos de desarrollo de capacidades de los actores y las instituciones educativas. Si bien los sistemas de accountability poseen este componente, su rol es menos protagónico que el que ha adquirido en países como Finlandia o Singapur.

Todo lo que se ha ido mencionado favorece concluir que las estrategias de accountability han resultado ser incompletas como mecanismos para propiciar el mejoramiento educativo. Debido a las características de este proceso, el estudio de los casos ayudó a comprender que las políticas educativas debieran promover el desarrollo de un sentido de responsabilización, junto con una dinámica de desarrollo de capacidades de mayor envergadura. Además, tienen que asegurar la existencia de plazos más amplios para observar el mejoramiento, y sobre todo destinar confianza en las capacidades de las escuelas y en las medidas que se tomen para fortalecerlas. Así, ha sido posible que algunos sistemas educativos hayan podido avanzar más allá de un espejismo de logro basado en mecanismos de control y de incentivos.

Recibido en 14 de febrero del 2014 Aprobado en 12 de julio del 2014

\section{Notas}

1 Pese a lo anteriormente señalado, se intentará describir de una forma que permita reconocer las otras tipologías de accountability.

2 La información para describir este caso se obtuvo de Ontario Ministry of Education (2012a; 2012b; 2012c), Education Quality and Accountability Office, EQAO

Educação \& Realidade, Porto Alegre, v. 40, n. 1, p. 275-298, jan./mar. 2015. 
Accountabilty y Mejoramiento Educativo

(2005a; 2005b; 2011; 2012; 2013), Ontario School Board Co-Ordinating Comitee (2012), Levin (2007), Volante e Jafaar (2008).

3 Ver Espínola \& Claro (2010)

4 Para describir este caso se recurrió a las siguientes fuentes: Ministerio de Educación Nacional (2009a; 2009b; 2010a; 2010b; 2013), Ley 1324 (2009), Banco Mundial (2009), ICFES (2013).

5 Lo anterior implica que, por ejemplo “[...] la denominación Desempeño Básico definida en la Escala de Valoración Nacional, lo que se está expresando es que tal educando ha aprobado y por tanto ha superado los parámetros fijados como básicos en los Estándares Básicos de Competencia, las Orientaciones Pedagógicas o Generales, según sea el caso. Por lo tanto, es inaceptable y éticamente poco responsable, adelantar procesos formativos por debajo de los parámetros fijados en los mencionado documentos" (Ministerio de Educación Nacional, 2009).

6 Para describir este caso, se utilizaron las siguientes fuentes: Ley 107-110 (2002), Departamento de Educación (2003), CEP (2005a; 2005b; 2007a; 2007b), Vazquez \& Darling-Hammond (2008), RAND (2009).

7 Para la descripción de este caso, se utilizaron las siguientes fuentes: Sahlberg (2010; 2011), Halivarvi et al. (2007), Ministry of Education and Culture (2013).

8 Para describir este caso se recurrió a las siguientes fuentes: Tan; Ng (2007), Ng (2010; 2012), Ministry of Education (2012a; 2012b), NIE (2012).

9 Para describir este caso, se recurrió a las siguientes fuentes: Ofsted (2012; 2013a; 2013b; 2013c), Preal (2011), West; Mattei; Roberts (2011).

\section{Referencias}

BANCO MUNDIAL. La Calidad de la Educación en Colombia. Un análisis y algunas opciones para un programa de política. Washington D.C., 200043 U.S.A. Banco Mundial, 2009.

BARBER, Michael. How Government, Professions and Citizens Combine to Drive Successful Educational Change. In: HARGREAVES, Andy; LIEBERMAN, Anne; FULLAN, Michael; HOPKINS, David (Ed.). Second International Handbook of Educational Change. Dordrecht, Heidelberg, London, New York: Springer Science+Business Media, 2010. v. 1. P. 261-278.

BELLEI, Cristián; CONTRERAS, Dante; VALENZUELA, Juan. Ecos de la Revolución Pingüina. Santiago: UNICEF, 2010.

BOGOTCH, Ira; MIRÓN, Luis; BIESTA, Gert. Effective for What; Effective for Whom? Two Questions SESI Should Not Ignore. In: TOWNSEND, Tony (Ed.). International Handbook of School Effectiveness and Improvement. Springer, 2007. v. 1. P. 93-110.

CENTER ON EDUCATIONAL POLICY. NCLB: Urban Schools Are Tagged for Improvement at Higher Rates Than Suburban or Rural. 2005a. Disponible en: <http://cep-dc.org/>. Extraído el: 06 mar. 2013.

CENTER ON EDUCATIONAL POLICY. NCLB: What School Districts Are Doing to Improve Teacher Quality in High-Need. 2005b. Disponible en: <http://cepdc.org/>. Extraído el: 06 mar. 2013.

CENTER ON EDUCATIONAL POLICY. No Child Left Behind at Five: A Review of Changes to State Accountability Plans. 2007a. Disponible en: <http://cep-dc. org/>. Extraído el: 06 mar. 2013. 
CENTER ON EDUCATIONAL POLICY. State Implementation of Supplemental Educational Services under the No Child Left Behind Act. 2007b. Disponible en: <http://cep-dc.org/>. Extraído el: 06 mar. 2013.

CENTER ON EDUCATIONAL POLICY. Some Perspectives from Rural School Districts on The No Child Left Behind Act. 2007c. Disponible en: <http://cepdc.org/>. Extraído el: 06 mar. 2013.

CLAD. CONSEJO LATINOAMERICANO DE ADMINISTRACIÓN PARA EL DESARROLLO. La Responsabilización (Accountability) en la Nueva Gestión Pública Latinoamericana. Caracas, Venezuela: CLAD, 2000.

DARLING-HAMMOND, Linda; ASCHER, Carol. Creating Accountability in Big City School Systems. Urban Diversity Series N. 102. 1991.

DARLING-HAMMOND, Linda. Estándares, Accountability y Reforma escolar. Teachers College Record, v. 106, n. 6, p. 1047-1085, June 2004.

DARLING-HAMMOND, Linda. Standards, Assesments, and Educational Policy: in pursuit of genuine accountability. Educational Testing Service, Princeton, NJ 08541-0001, 2006.

DARLING-HAMMOND, Linda. Teaching and Educational Transformation. In: HARGREAVES, Andy; LIEBERMAN, Ann; FULLAN, Michael; HOPKINS, David (Ed.). Second International Handbook of Educational Change. Dordrecht, Heidelberg, London, New York: Springer Science+Business Media, 2010. v. 1. P. 505520.

DEPARTAMENTO DE EDUCACIÓN DE ESTADOS UNIDOS. Que Ningún Niño se Quede Atrás. Guía para padres. Washington D.C., 2003.

DI GROPELLO, Emanuella. La Descentralización de la Educación y las Relaciones de Rendición de Cuentas en los Países Latinoamericanos. Documento de Trabajo, 30. 2004.

ELMORE, Richard. Mejorando la Escuela desde la Sala de Clases (C. Santa Cruz, Trans.). Santiago: Fundación Chile, Fundación CAP, 2010.

EQAO. Educational Quality and Accountability Office. Working Together for Student Success. Ontario, Toronto, 2005a.

EQAO. Educational Quality and Accountability Office. EQAO Guide to School and Board Improvement Planning. Ontario, Toronto, 2005b.

EQAO. Educational Quality and Accountability Office. Improving Education Through Evidence of Learning. Ontario, Toronto, 2011.

EQAO. Educational Quality and Accountability Office. The Power of Ontarioss Provincial testing Program. Ontario, Toronto, 2012.

EQAO. Educational Quality and Accoutability Office. Working together for Student Success. A School improvement Planning Guide. Ontario, Toronto, 2013.

ESPÍNOLA, Viola; CLARO, Juan. El Sistema Nacional de Aseguramiento de la Calidad: una reforma basada en estándares. In: CRISTIÁN, Bellei; CONTRERAS, Dante; VALENZUELA, Juan (Ed.). Ecos de la Revolución Pingüina. Avances, debates y silencios en la reforma educacional. Santiago: Universidad de Chile, 2010. P. 51-83.

FULLAN, Michael. The Meaning of Educational Change. New York: Teachers College Press, 1982.

FULLAN, Michael. Los Nuevos Significados del Cambio en la Educación. Barcelona: Octaedro, 2002.

Educação \& Realidade, Porto Alegre, v. 40, n. 1, p. 275-298, jan./mar. 2015. 
Accountabilty y Mejoramiento Educativo

FULLAN, Michael. Investigación sobre el Cambio Educativo: presente y futuro. Revista Digital de Investigación Lasaliana, v. 3, p. 31-35, 2011.

GUHN, Martin. Insights from Successful and Unsuccessful Implementations of School Reform Programs. Journal of Educational Change, v. 10, p. 337-363, 2011. HALIJARVI, Jouni; KUPARI, Pekka; LINNAKYLA, Pirjo; REINIKAINEN, Pasi; SULKUNEN, Sari; TORNROO, Jukka; ARFFMAN, Inga. The Finnish Success in PISA and some Reasons behind it. Institute of Educational Research. Helsinki University: Helsinki, 2007.

HALLINGER, Phillip; HECK, Ronald. Exploring the journey of school improvement: classifying and analyzing patterns of change in school improvement processes and learning outcomes, School Effectiveness and School Improvement. International Journal of Research, Policy and Practice, v. 22, n. 1, p. 1-27, 2011.

HARGREAVES, Andy. Enseñar en la Sociedad del Conocimiento. Barcelona: Octaedro, 2003.

HARGREAVES, Andy. Change from Without: Lessons from Other Countries, Systems, and Sectors. In: HARGREAVES, Andy et al. (Ed.). The Second International Handbook of Educational Change, Springer International Handbooks of Education, Dordrecht, Springer, 2010. V. 23. P. 105-117.

HARGREAVES, Andy; FINK, Dean. Estrategias de cambio y mejora en educación caracterizadas por su relevancia, difusión y continuidad en el tiempo. Revista de Educación, n. 339, p. 43-58, 2006.

HARGREAVES, Andy; SHIRLEY, Dennis. La Cuarta Vía. El prometedor futuro del cambio educativo. Barcelona: Octaedro, 2012.

HOPKINS, David. Hacia una Buena Escuela: experiencias y lecciones (C. Santa Cruz, Trans.). Santiago: Fundación Chile, Fundación CAP, 2008.

ICFES. Instituto Colombiano para la Evaluación de la Educación. Informe de Rendición de cuentas 2013. 2013. Disponible en: <www.igfes.gov.co/el-icfes-rinde-cuentas>. Extraído el: 30 dec. 2013.

LEVIN, Ben. Sustainable, large-scale education renewal. Journal Education Change, v. 8, p. 323-336, 2007. DOI 10.1007/s10833-007-9041.

LEVIN, Ben. How to Change 5000 Schools: A practical and positive approach for leading change at every level. Cambridge, MA: Harvard Education Press, 2008.

LEVIN, Ben. Does politics help or hinder education change? Journal of Educational Change, v. 10, p. 69-72, 2009.

MARTINIC, Sergio; ELACQUA, Gregory (Ed.). ¿Fin de Ciclo? Cambios en la gobernanza del sistema educativo. Santiago: UNESCO, 2010.

MCMEEKIN, Robert. Hacia una comprensión de la accountability educativa y cómo puede aplicarse en los países de América Latina. In: CORVALÁN, Javier (Ed.). Accountability Educacional: posibilidades y desafíos para América Latina a partir de la experiencia internacional. Santiago: Cide - Preal, 2006. P. 19-47.

MINISTERIO DE EDUCACIÓN NACIONAL. Fundamentaciones y Orientaciones para la Implementación del Decreto 1290 de 2009. Bogotá, 2009a.

MINISTERIO DE EDUCACIÓN NACIONAL. Decreto 1290 de 2009. Bogotá, 2009b.

MINISTERIO DE EDUCACIÓN NACIONAL. Educación de Calidad, el Camino para la Prosperidad. Bogotá, 2010.

MINISTERIO DE EDUCACIÓN NACIONAL. Plan de Acción 2013. Bogotá, 2013. 
MINISTRY OF EDUCATION AND CULTURE OF FINLAND. Brochure 2013. 2013. Disponible en: <http://www.minedu.fi/export/sites/default/OPM/Julkaisut/2013/liitteet/Brochure_2013.pdf?lang=en>. Extraído el: 30 mayo 2013.

MINISTRY OF EDUCATION OF SINGAPORE. The Desired Outcomes of Education. 2012a. Disponible en: <www.moe.gov.sg>. Extraído el: 25 mayo 2013.

MINISTRY OF EDUCATION OF SINGAPORE. Secondary School Education. 2012b. Disponible en: <www.moe.gov.sg>. Extraído el: 25 mayo 2013.

MINISTRY OF EDUCATION AND CULTURE OF FINLAND. Organic Educational Act (628). Finland, 1998

MINISTRY OF EDUCATION AND CULTURE OF FINLAND. Brochure 2013. 2013. Disponible en: <http://www.minedu.fi/export/sites/default/OPM/Julkaisut/2013/liitteet/Brochure_2013.pdf?lang=en.> Extraído el: 30 mayo 2013.

MÖLLER, Jorunn. School Leadership in an Age of Accountability: tensions between managerial and professional accountability. Journal of Educational Change, v. 10, p. 37-46, 2009. DOI 10.1007/s10833-008-9078-6.

MÜLLER, Jorg; HERNÁNDEZ, Fernando. On the Geography of Accountability: comparative analysis of teachers' experiences across seven European countries. Journal of Educational Change, v. 11, p. 307-322, 2010. DOI 10.1007/s10833-009-9126-x.

MURILLO, Francisco. El Movimiento Teórico-práctico de Mejora en la Escuela. Algunas lecciones. Revista Electrónica Iberoamericana sobre Calidad, Eficacia y Cambio en Educación, v. 2, n. 1, p. 1-22, 2003.

MURILLO, Francisco; KRICHESKY, Gabriela. El Proceso de Cambio Escolar. Una Guía para Impulsar y Sostener la Mejora de las Escuelas. Revista Iberoamericana sobre Calidad, Eficacia y Cambio en Educación, v. 10, n. 1, 2012. P. $27-43$. NG, Pak Tee. The Evolution and Nature of Educational Accountability in Singapore Educational System. Education Assessment, Evaluation and Accountability, v. 22, p. 275-292, 2010.

NG, Pak Tee. An examination on school accountability from the perspective of Singapore School Leaders. Educational Research Policy and Practice, 2012.

NIE. National Institute of Education of Singapore. PISA. Lessons for and from Singapore. CJ Koh Proffesioral Lecture Series N² 2. NIE/ NTU/ Singapore. 2012.

OFSTED. Office for Standards in Education Children Services and Skills. Annual Report and Accounts 2011-2012. Londres, 2012.

OFSTED. Office for Standards in Education Children Services and Skills. A Good Education for All: Inspections of Local authority services. Londres, 2013a.

OFSTED. Office for Standards in Education Children Services and Skills. The Framework for the Inspection of Local Authorities. Londres, 2013b.

ONTARIO MINISTRY OF EDUCATION. Education Labour Update. What do you need to know about effective uses of diagnostics. 2012a. Disponible en: <www. edu.gov.on.ca/en>. Extraido el: 5 mayo 2013.

ONTARIO MINISTRY OF EDUCATION. Education Labour Update. What do you need to know about fair hiring. 2012b. Disponible en: <www.edu.gov.on.ca/en>. Extraído el: 5 mayo 2013.

ONTARIO SCHOOL BOARD CO-ORDINATING COMITEE. Putting Students First Act, 2012. Ontario, Toronto, 2012.

PREAL. Programa de Promoción de la Reforma Educativa en América Latina. Estrategias de Apoyo a Escuelas para Mejorar los Aprendizajes. Experiencias en Inglaterra y Canadá. Serie Mejores Prácticas, año 13, v. 38, 2011.

Educação \& Realidade, Porto Alegre, v. 40, n. 1, p. 275-298, jan./mar. 2015. 
RAND Corporation. State and Local Implementation of the No Child Left Behind Act. Volume VI - Targeting and Uses of Federal Education Funds. Washington D.C., 2009

REPÚBLICA DE COLOMBIA. Gobierno Nacional. Ley n. 1324. Parámetros del Sistema de Evaluación Nacional. Colombia, 2009.

SAHLBERG, Passi. Rethinking Accountability in a Knowledge Society. Journal of Educational Change, v. 11, p. 45-61, 2010.

SAHLBERG, Passi. The Forth Way of Finland. Journal of Educational Change, v. 12, p. 173-185, 2011.

TAN, Charlene; NG, Pak. Dynamics of Change. Decentralised centralism of education in Singapore. Journal of Educational Change, v. 8, p. 155-168, 2007.

UNITED STATES OF AMERICA CONGRESS. Public Law 107-110. No Child Left Behind. Washington D.C., 2002.

VASQUEZ, Julian; DARLING-HAMMOND, Linda. Accountability Texas-Style: The Progress and Learning of Urban Minority Students in a High-Stakes Testing Context. Educational Evaluation and Policy Analysis, v. 30, n. 2, p. 75-110, jun. 2008.

VOLANTE, Louis; JAAFAR, Sonia. Educational assessment in Canada. Assessment in Education: Principles, Policy \& Practice, v. 15, n. 2, p. 201-210, 2008.

WEINSTEIN, José; MUÑOZ, Gonzalo (Ed.). ¿Qué Sabemos sobre los Directores de Escuela en Chile? Santiago: Fundación Chile y P. Universidad Católica de Chile, 2012.

WEST, Anne; MATTEI, Paola; ROBERTS, Jonathan. Accountability and Sanctions in British Schools. British Journal of Educational Studies, v. 59, n. 1, p. 41-62, 2011.

Luis Felipe de la Vega Rodríguez es sociólogo, magíster en política y gobierno, magíster en Ciencias de la Educación, Doctor (c) en Ciencias de la Educación.

E-mail: ldelaveg@uc.cl 